\section{Correlation between mean platelet volume and total risk of cardiovascular disease}

\author{
DOMAGOJ MARKOVIĆ • VEDRAN CAREVIĆ • DAMIR BONACIN • \\ BRANKA PAUKOVIĆ SEKULIĆ • ADA SAPUNAR • DAMIR FABIJANIĆ
}

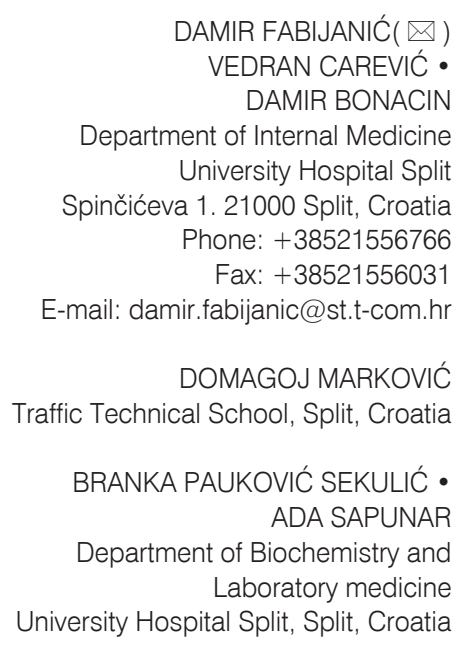

DAMIR FABIJANIĆ $(\varangle)$ VEDRAN CAREVIĆ • DAMIR BONACIN Department of Internal Medicine University Hospital Split Spinčićeva 1. 21000 Split, Croatia Phone: +38521556766 Fax: +38521556031 E-mail: damir.fabijanic@st.t-com.hr

DOMAGOJ MARKOVIĆ Traffic Technical School, Split, Croatia BRANKA PAUKOVIĆ SEKULIĆ • ADA SAPUNAR

Department of Biochemistry and Laboratory medicine University Hospital Split, Split, Croatia

\begin{abstract}
Introduction. The association between mean platelet volume (MPV) and single cardiovascular disease (CVD) risk factors has previously been tested. The aim of our study was to evaluate the association between MPV and total risk of CVD. Materials and Methods. In 216 consecutive outpatients referred for a cardiology assessment because of multiple CVD risk factors, total CVD risk was estimated according to the Framingham Risk Score (FRS) as low (<10\%), intermediate (11-19\%), and high (>20\%). Additionally, FRS was adjusted for triglycerides (TG), body mass index (BMI) and a family history of CVD, important CVD risk factors which are not included in the FRS equation. Also, CVD risk was estimated according to terciles of $M P V(<8.3,8.3-9.1,>9.1 \mathrm{fL}$, respectively). Correlation between high sensitivity C-reactive protein (hs-CRP) and FRS was used as confirmation that our study hypothesis was properly set.

Results. MPV did not correlate with individual CVD risk factors or with FRS, either before, or after adjustment for additional CVD risk factors. Significant correlation between MPV and total CV risk was observed only in the adjusted high FRS group; namely, for persons in tercile 3, CVD risk was doubled (OR 2.2; 95\% Cl 1.05-4.64; $P=0.038$ ). In comparison, there was a strong linear correlation between $h s-C R P$ and unadjusted $(r=0.203, P=0.004)$ and adjusted $(r=0.383, P<0.001) F R S$.

Conclusion. Correlation between MPV and high total CVD risk suggests increased platelet activity primarily as a consequence and sign of atherosclerotic vascular damage. Linear correlation between hs-CRP with FRS confirms inflammation as an important mechanism of atherogenesis.
\end{abstract}

Key words: platelets, atherosclerosis, coronary risk prediction, Framingham risk score.

\section{Introduction}

Major cardiovascular and cerebrovascular events (e.g. myocardial infarction and stroke) can occur in individuals without known pre-existing cardiovascular (CV) disease. Because CV diseases are more likely to occur in patients with certain risk factors, prevention of such events, including the accurate identifi- cation of those at increased risk, has been developed using traditional CV risk factors (e.g. cigarette smoking, blood pressure, cholesterol, diabetes mellitus, obesity, family history of CV diseases). The coexistence of multiple $\mathrm{CV}$ risk factors multiplies the risk of adverse CV events. (1) Following this, total CV risk assessment using models that include multiple $\mathrm{CV}$ risk factors is recommended. One of these models is the Framingham Risk Score (FRS), a simplified prediction tool developed to enable clinicians to estimate CV risk in individuals, often considered the reference standard. (2) It includes age, gender, smoking, blood pressure, total and HDL-cholesterol values, and diabetes, and estimates the 10 year risk of CV events by stratifying individuals into the three risk categories: low, intermediate, and high. (2)

In order to better assess CV risk and to clarify the pathogenesis of atherothrombosis, some biochemical markers are stressed. One of them is mean pla- 
telet volume (MPV), a potential marker of platelet reactivity, which is one of the major culprit mechanisms in atherothrombotic events. Large platelets - characterized by a higher MPV - contain more dense granules and are metabolically and enzymatically more active than small platelets; therefore these platelets have higher thrombotic potential. Elevated values of MPV are associated with $\mathrm{CV}$ diseases, and also predict a poor outcome following acute coronary events or coronary interventions. (3)

Several reports have demonstrated an association between MPV and traditional CV risk factors, such as hypertension, impaired fasting glucose, diabetes, hypercholesterolemia and obesity, suggesting a possible mechanism in increasing CV risk. (4-8) Based on these proven associations between MPV and single CV risk factors, in light of recommendations for total CV risk assessment, (1) we assessed the association between MPV and total CV risk.

\section{Materials and Methods}

\section{Subjects}

In this study we enrolled 216 consecutive subjects [146 (65.74\%) males], all older than 18 years of age (median age 65 (26-87) years), without a history of clinically apparent CV diseases, that had been admitted to the Cardiology Department at the University Hospital of Split due to multiple CV risk factors. Recent research has shown that rosuvastatin and losartan can interfere with MPV so patients who used them were exluded from our study to eliminate possible errors. Exclusion criteria consisted of a previous diagnosis of CV diseases (coronary artery disease, history of myocardial infarction or stroke, chronic heart failure), thyroid disease, renal or hepatic dysfunction, hematological disease, cancer, thrombocytopenia, autoimmune disease, systemic inflammatory conditions, pregnancy, use of estrogen therapy (oral contraceptives or hormone replacement therapy) or serotonin reuptake inhibitor drug use.

The study was conducted according to the principles of the Helsinki Declaration. The local Ethics Committee gave approval for this study. All patients agreed to participate voluntarily in the study after a detailed explanation of study objectives. All participants provided written informed consent after detailed explanation of study objectives.

\section{Collection of patient data}

Trained research assistants filled out the questionnaire on demographic characteristics (age, sex, height and weight), the presence of traditional CV risk factors (arterial hypertension, diabetes, hypercholesterolemia, hypertriglyceridemia, cigarette smoking, family history of CV diseases), and medication used before admission.

The presence of $\mathrm{CV}$ risk factors was defined as following: (1) diabetes mellitus - fasting blood glucose $>6.9$ $\mathrm{mmol} / \mathrm{L}$ or non-fasting glucose level $>11 \mathrm{mmol} / \mathrm{L}$, or use of antidiabetic medication. The subjects were identified as either presently suffering from diabetes or not; (2) arterial hypertension - systolic blood pressure $>140 \mathrm{~mm}$ $\mathrm{Hg}$, diastolic blood pressure $>90 \mathrm{~mm}$ $\mathrm{Hg}$, or previous use of antihypertensive drugs; (3) hypercholesterolemia - total cholesterol (TC) $>4.5 \mathrm{mmol} / \mathrm{L}$, LDLcholesterol (LDL-C) $>2.5 \mathrm{mmol} / \mathrm{L}$, or previous use of lipid-lowering therapy; (4) hypertriglyceridemia - triglycerides (TG) $>1.8 \mathrm{mmol} / \mathrm{L}$; (5) overweight body mass index (BMl: weight/height ${ }^{2}$ ) $>25 \mathrm{~kg} / \mathrm{m}^{2}$; (6) cigarette smoking was categorized as non smoking or ever smoking; (7) family history of CV diseases included a history of sudden death, myocardial infarction, angina pectoris or coronary revascularization in a first relative before the age of 55 years (men) or 65 years (women).

Collection and processing of diagnostic blood specimen

Blood samples were collected in the morning after a 20-min rest following a fasting period of 12 hours for the measurement of TC, LDL-C, HDL-cholesterol (HDL-C), TG, and serum glucose. Plasma concentrations of glucose, TG, TC and HDL-C (after selective precipitation with Immuno AG, Vienna, Austria) were measured by standard enzymatic methods on an Olympus AU-640 (Olympus, Tokyo, Japan). LDL-C was calculated using the Friedwald equation. If TG concentration was above 3 $\mathrm{mmol} / \mathrm{L}, \mathrm{HDL}-\mathrm{C}$ and LDL-C were measured by direct immunoinhibition method (Olympus Diagnostica, Lismeehan, Ireland) and homogeneous assay (Randox Laboratories, Crumlin, United Kingdom), respectively.

MPV was measured within 30 minutes of sampling by Bechman Counter (USA) in a blood sample collected in citrate $(1: 4 \mathrm{v} / \mathrm{v})$ in order to avoid platelet swelling induced by EDTA. Normal range of MPV in our laboratory ranged from 6-10.8 fL.

Assessment of individual's CV disease risk level

Eight risk factors (age, sex, systolic and diastolic blood pressure, cholesterol and HDL-C levels, smoking, diabetes) were used to calculate an individual's risk level of future development of CV diseases based on the FRS. Among these factors, age, blood pressure, cholesterol and HDL-C levels were processed according to their values and sex, smoking and diabetes were processed depending on whether it was positive or negative. Using these risk factors, the 10-year predicted risk of developing manifest CV disease was calculated for each case. In accordance with the FRS, the study population was then categorized as low $(\leq 10 \%)$, intermediate $(11-19 \%)$, and high ( $\geq 20 \%)$ risk. After that, in order to better estimate total CV risk, FRS was adjusted for TG, BMI and family history of $\mathrm{CV}$ diseases, traditional CV risk factors that are not included in the FRS equation.

High sensitivity C-reactive protein (hsCRP) as a control

Hs-CRP, widely accepted as an independent predictor of CV diseases, significantly correlates with calculated 10-year FRS. (9) Therefore, we tested the correlation between hs-CRP and FRS as a confirmation that our study hypothesis was properly set.

Hs-CRP determination was performed with high sensitivity immunoturbidimetric assay (Cobas Integra 700, Roche, 
Table 1. Study sample characteristics.

\begin{tabular}{|c|c|c|c|c|c|}
\hline & \multirow{3}{*}{$\begin{array}{c}\text { Total } \\
\mathrm{N}=216\end{array}$} & \multicolumn{3}{|c|}{ FRS (\%) } & \multirow[b]{3}{*}{$\mathrm{P}$} \\
\hline & & $\leq 10$ & $11-19$ & $\geq 20$ & \\
\hline & & $N=29(13 \%)$ & $\mathrm{N}=59(27 \%)$ & $\mathrm{N}=128(60 \%)$ & \\
\hline \multicolumn{6}{|c|}{ Demographic parameters and $\mathrm{CV}$ risk factors } \\
\hline FRS (\%) & $23.8 \pm 11.9$ & $13 \pm 7.2$ & $28.4 \pm 14.3$ & $58.6 \pm 12.8$ & $<0.001$ \\
\hline Male (\%) & 65.74 & 7.7 & 24.6 & 67.6 & $<0.001$ \\
\hline Age (years; $M \pm S D$ ) & $65 \pm 9.7$ & $56.78 \pm 12.06$ & $63.59 \pm 8.71$ & $68.3 \pm 8.24$ & $<0.001$ \\
\hline $\mathrm{BMI}\left(\mathrm{kg} / \mathrm{m}^{2} ; \mathrm{M} \pm \mathrm{SD}\right)$ & $28.7 \pm 3.7$ & $26.6 \pm 4.06$ & $27.26 \pm 3.61$ & $28.7 \pm 3.57$ & 0.004 \\
\hline Overweight and obese (\%) & 82.2 & 10.3 & 25.3 & 64.4 & $<0.001$ \\
\hline Arterial hypertension (\%) & 72.6 & 9.7 & 24.5 & 65.8 & $<0.001$ \\
\hline Systolic BP (mm Hg, M \pm SD) & $138.5 \pm 19.3$ & $123.04 \pm 12.12$ & $131.72 \pm 16.04$ & $145.21 \pm 19.11$ & $<0.001$ \\
\hline Diastolic BP (mm Hg, M $\pm \mathrm{SD}$ ) & $82.9 \pm 11$ & $79.11 \pm 9.53$ & $80.59 \pm 10.68$ & $84.94 \pm 11.04$ & 0.005 \\
\hline Diabetes mellitus (\%) & 27.8 & 1.7 & 16.7 & 49.81 & $<0.001$ \\
\hline Hypercholesterolemia (\%) & 27 & 13. 9 & 27.8 & 91.63 & $<0.001$ \\
\hline Hypertriglyceridemia (\%) & 50.2 & 0 & 24.7 & 75.3 & $<0.001$ \\
\hline Cigarette smoking (\%) & 13.4 & 10.3 & 20.7 & 69 & $<0.001$ \\
\hline Family history of CVD (\%) & 14.8 & 8.4 & 11.2 & 26.3 & 0.07 \\
\hline \multicolumn{6}{|l|}{ Laboratory parameters $(\mathrm{M} \pm \mathrm{SD})$} \\
\hline Glucose (mmol/L) & $6.7 \pm 4.5$ & $5.47 \pm 0.68$ & $6.15 \pm 1.73$ & $7.32 \pm 5.69$ & 0.067 \\
\hline Total cholesterol (mmol/L) & $5 \pm 1.3$ & $4.43 \pm 1$ & $4.9 \pm 1.21$ & $5.25 \pm 1.06$ & 0.004 \\
\hline LDL-cholesterol (mmol/L) & $2.8 \pm 0.97$ & $2.42 \pm 0.9$ & $2.78 \pm 0.96$ & $3.2 \pm 1.06$ & 0.001 \\
\hline HDL-cholesterol (mmol/L) & $1.36 \pm 0.4$ & $1.51 \pm 0.36$ & $1.5 \pm 0.47$ & $1.25 \pm 0.32$ & 0.001 \\
\hline Triglycerides (mmol/L) & $1.8 \pm 1.8$ & $1.1 \pm 0.34$ & $1.55 \pm 2.95$ & $2.15 \pm 2.2$ & 0.005 \\
\hline hs-CRP (mg/L) & $2.46 \pm 2.9$ & $1.7 \pm 1.72$ & $2.4 \pm 2.95$ & $3.3 \pm 4.08$ & 0.064 \\
\hline MPV (fL) & $8.77 \pm 1.03$ & $8.69 \pm 1.18$ & $8.78 \pm 1.14$ & $8.84 \pm 1.07$ & 0.79 \\
\hline \multicolumn{6}{|c|}{ Medications before admission (\% of pts.) } \\
\hline Acetylsalicylic acid & 57.9 & 15.2 & 26.4 & 58.4 & 0.473 \\
\hline Beta blockers & 69 & 12.7 & 26.7 & 60.7 & 0.632 \\
\hline Calcium channel blockers & 16.7 & 11.1 & 16.7 & 72.2 & 0.167 \\
\hline ACE inhibitors & 47.22 & 4.9 & 31.4 & 63.7 & 0.003 \\
\hline Statins & 54.2 & 16.2 & 32.5 & 51.3 & 0.052 \\
\hline
\end{tabular}

BMI, body mass index; BP, blood pressure; FRS, Framingham Risk Score; CVD, cerebrovascular disease; hs-CRP, high sensitivity C-reactive protein; MPV, mean platelet volume.

Table 2. Correlation between mean platelet volume (MPV) and cardiovascular risk factors.

\begin{tabular}{lcc}
\hline & $\mathrm{R}^{*}$ & $\mathrm{P}$ \\
\hline Age & 0.01 & 0.927 \\
Body Mass Index & -0.03 & 0.692 \\
Systolic blood pressure & 0.08 & 0.261 \\
Diastolic blood pressure & 0.09 & 0.175 \\
Total cholesterol & -0.01 & 0.424 \\
Triglycerides & -0.07 & 0.353 \\
HDL cholesterol & 0.1 & 0.156 \\
Fasting plasma glucose & 0.01 & 0.926 \\
Current smoking & -0.05 & 0.429 \\
High sensitivity C-reactive protein & 0.01 & 0.936 \\
\hline
\end{tabular}

Mannheim, Germany) with a detection limit of $0.25 \mathrm{mg} / \mathrm{L}$, at the time of admission and in a blind fashion.
Statistical analysis

Calculation of differences between baseline characteristics is presented as a means and standard deviation $(\mathrm{M} \pm \mathrm{SD})$ or percentage frequencies. Normally distributed values were compared with Student's tests, and skewed continuous variables by non-parametric rank sum tests. Chi-square tests or Fisher's exact test were used for categorical variables. Pearson's correlation was used to evaluate the association between MPV and serum values for single CV risk factors (age, sex, serum glucose, TC, HDL-C, LDL-C, systolic and diastolic blood pressure, BMI, smoking). The same test was used in assessment of the correlation between MPV and total CV risk expressed as FRS before and after adjusting for multiple potential confounders such as TG, $\mathrm{BMI}$ and a family history of CV disease. 


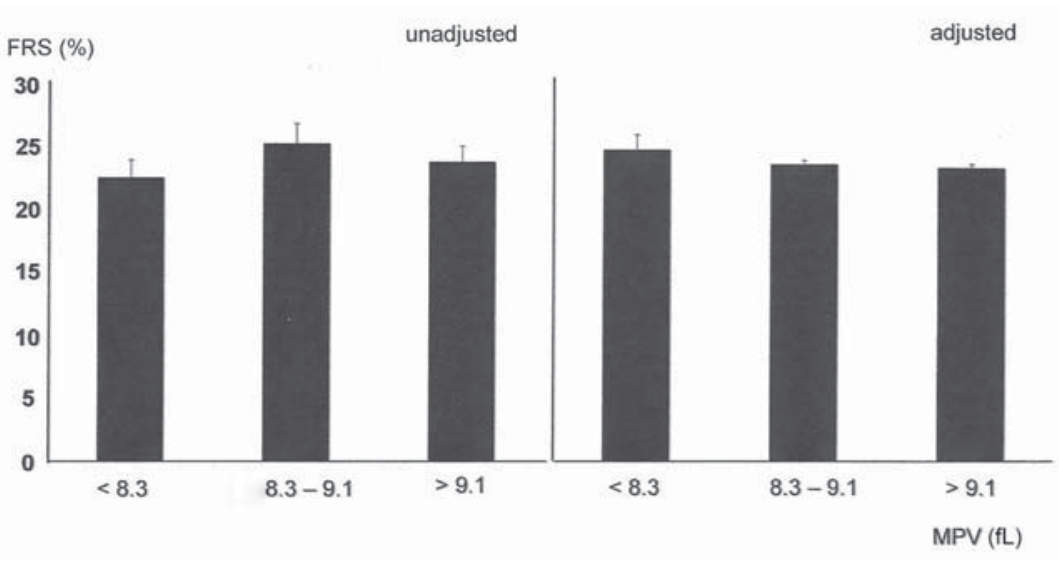

Figure 1. Correlation between MPV and unadjusted and adjusted (adjusted for triglicerydes, obesity, and family history of cardiovascular disease) Framingham Risk Score (FRS).

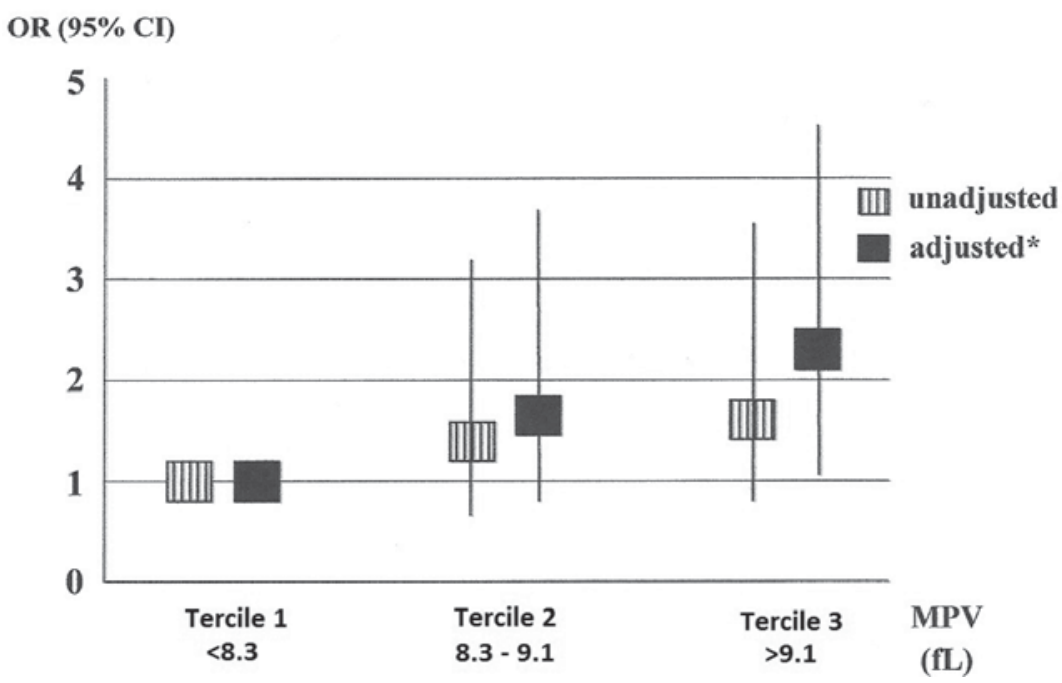

Figure 2. Correlation between C-reactive protein and unadjusted and adjusted (adjusted for triglicerydes, obesity, and family history of cardiovascular disease) Framingham Risk Score (FRS).

The ANOVA one way analysis was used to test the difference in the FRS among terciles of MPV (tercile 1, <8.3 fL; tercile 2, 8.3-9.1 fL; and tercile 3, >9,1 fL) followed by multiple comparisons with the Bonferroni correction. Thereafter, an analysis of covariance was performed with the above-listed confounders as covariates. A multiple logistic regression analysis was performed to determine the odds ratio (OR) of high-risk for CV disease in each tertile of MPV, before and after adjusting for TG, BMI, and family history of CV disease. All statistical tests were two-sided and a
P-value of less than 0.05 was considered to be significant.

All analyses were performed with the Statistica 10 software package (StatSoft. Inc., Tulsa, USA).

\section{Results}

The baseline characteristics of the study population by FRS are presented in table 1. The mean FRS in the study subjects was $23.8 \pm 11.9 \%$; 29 (13\%) of the subjects were in the low risk (FRS <10\%), 59 (27\%) were in the intermediate risk (FRS $11-19 \%)$, and 128 (60\%) were in the high risk (FRS > 20\%) group.
The median age of the whole study population was 65 (26-87) years. The most commonly represented risk factors were overweight (82.2\%), hypertension $(72.6 \%)$ and hypertriglyceridemia (50.2\%), followed by diabetes (27.8\%), hypercholesterolemia (27\%), family history for CV diseases (14.8\%) and smoking (13.4\%).

The frequency and values of all CV risk factors were significantly increased from lower to higher FRS group (table 1). Both, MPV $(P=0.79)$ and hs-CRP $(P=0.064)$ values showed a nonsignificant increase according to FRS category.

A significantly higher percentage of high-risk patients used CV medications (table 1). However, a difference was only observed in relation to the use of ACE-inhibitors $(P=0.003)$. The difference in statins use was on the border of statistical significance ( $P=0.052$ ).

Pearson's correlation coefficients of MPV with several clinical parameters are shown in table 2. There was no significant correlation between MPV and individual CV risk factors. A borderline, but not statistically significant correlation was observed between MPV and systolic blood pressure $(r=0.078$, $P=0.261)$, diastolic blood pressure $(r=0.094, P=0.175)$ and HDL-C $(r=0.099, P=0.156)$. There was no significant correlation between MPV and FRS $(r=0.008, P=0.914)$. Furthermore, the correlation remained insignificant after adjusting for TG, BMI, and family history of CV disease (partial $r=-0.059$, $P=0.397)$. Also, there was no significant correlation between MPV and hsCRP $(r=0.006, P=0.936)$.

There was a strong linear correlation between hs-CRP and FRS before ( $r=0.203, P=0.004)$ and after adjusting for TG, BMI, and a family history of CV disease $(r=0.383, P<0.001)$ (figure 2).

Although the FRS in the tercile 2 of MPV was slightly higher than in the tercile 1 and tercile 3 , a statistically significant difference in unadjusted FRS values among MPV terciles was not revealed $(F=0.93, d f=2, P=0.398)$ (figure 1 , left panel). Correlation between FRS, 
according to terciles of MPV after adjusting for TG, BMI, and family history for $\mathrm{CV}$ diseases, is presented in figure 1, right panel. Paradoxically, the FRS values were slightly decreased from MPV tercile 1 to MPV tercile 3. However, even after adjusting differences in FRS, correlation among MPV terciles were remained nonsignificant $(F=1.16$, $\mathrm{df}=2, \mathrm{P}=0.316$ ).

The ORs of high-risk for CV disease (FRS $>20 \%$ ) in each tercile of MPV are presented in figure 3 . Insignificant increases in unadjusted risk in each tercile were noted; OR for tercile 2 (regarding to the referent tercile 1) was 1.56 (95\% Cl 0.79-3.06; $P=0.19$, and for tercile 3 was 1.73 (95\% Cl 0.88-3.42; P $=0.11$ ). Significant correlation between MPV and total CV risk was observed only in the adjusted high FRS group. Namely, after adjusting for TG, BMI, and a family history of CV disease, for persons in tercile 3, a doubled increase in CV risk (OR 2.2; 95\% Cl 1.05-4.64; $\mathrm{P}$ $=0.038$ ) was noted in comparison to tercile 1.

\section{Discussion}

Although the association between MPV and single traditional CV risk factors has previously been tested, to the best of our knowledge this study is the first to investigate the association between MPV and total individual CV risk. Contrary to most previous studies, (3-8) our study did not demonstrate an association between MPV and single traditional $\mathrm{CV}$ risk factors. Moreover, correlation between MPV and total CV risk became significant only in patients with high FRS after adjusting for TG, BMI and family history for CV diseases, important traditional risk factors which are not included in the FRS equation.

According to current theories, atherosclerosis is a chronic inflammatory process in which many cell types participate in its initiation and progression. (10) Traditional CV risk factors damage endothelial cells and initiate atherosclerotic processes for which progression is an important interaction between activated endothelial cells, platelets, monocytes, macrophages,

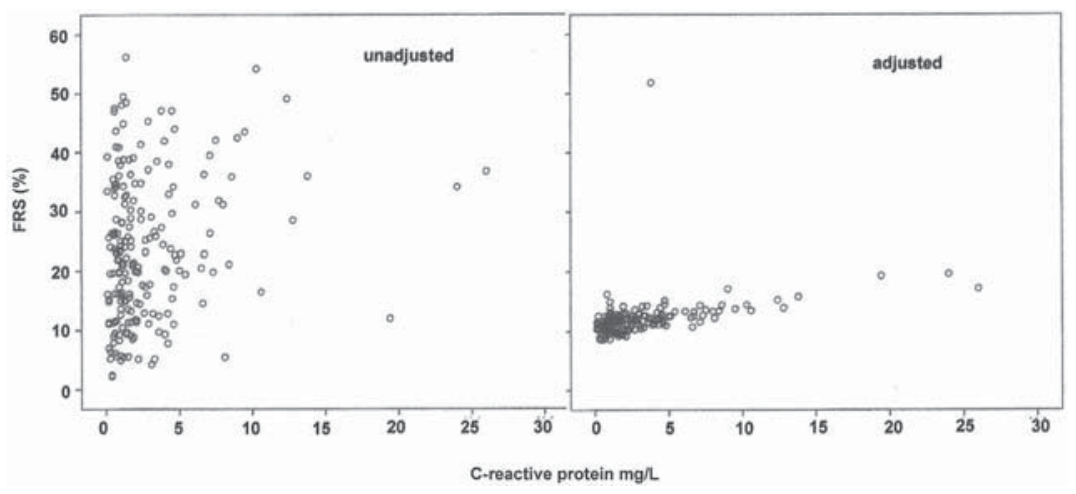

FRS, Framingham Risk Score; MPV, mean platelet volume; OR, odds ratio.

\section{Figure 3. Increasing risk for high-risk FRS (>20\%) group based on MPV; adjusted for triglyceridemia, body mass index and family history of cardiovascular disease.}

and smooth muscle cells. It is likely that platelets represent an important linkage between inflammation, atherogenesis and thrombosis. (10-12)

Increased platelet activity is associated with increased platelet volume. (3) Large platelets that contain more dense granules are metabolically and enzymatically more active than small platelets and containing more prothrombotic material. $(3,12,13)$ They show greater aggregability in response to ADP and decreased inhibition of aggregation by prostacyclin in vitro. Large platelets are denser and contain more $\alpha$-granules which can release prothrombotic substances. $(3,12,13)$ Based on these observations, elevated MPV is accepted as an indicator of platelet activation and indirectly, as a marker of increased CV risk.

A close relationship between MPV and CV risk factors, such as hypertension, impaired fasting glucose, diabetes, hypercholesterolemia and obesity has been demonstrated in several studies. (3-8) In hypertension and diabetes, MPV is higher in patients with the progression of basic disease and the presence of target organ damage. $(14,15)$ In patients with diabetes, MPV positively correlates with microvascular complications, and severity of angiographically proven coronary artery disease. (14) In hypertensive patients, MPV levels strongly correlate with severity of subclinical target organ dama- ge, including carotid atherosclerosis, left ventricular hypertrophy and renal dysfunction. (15)

Hypercholesterolemia, hypertriglyceridemia and abdominal obesity are directly associated with elevated MPV. (16) Moreover, when hypertriglyceridemia and abdominal obesity combine with elevated blood pressure and hyperglycemia, MPV elevation becomes more expressed and dependent on metabolic status. (17)

Observations that MPV positively correlates with single traditional risk factors and that the coexistence of multiple $\mathrm{CV}$ risk factors multiplies the risk of adverse CV events, formed the basis of our hypothesis that MPV positively correlates with total CV risk. Following this, we performed total CV risk assessment using the FRS, a simplified prediction tool developed to enable clinicians to estimate an individual's CV risk, and often considered the reference standard. (2) Unexpectedly, we did not reveal a linear association between MPV and FRS. The correlation between MPV and FRS became significant only in people with a high FRS after adjusting for TG, BMI, and family history of $\mathrm{CV}$ disease. A possible explanation is that being overweight and having hypertriglyceridemia, important factors for metabolic syndrome (16-18), were more frequently observed in patients in the high FRS group. Therefore, our results stress the importance of these 
factors in atherogenesis and also in CV risk prediction. Namely, the FRS does not account for metabolic syndrome which is strongly associated with atherogenesis, and often underestimates total CV risk in patients with these risk factors. (16-18)

Correlation between MPV and high total CV risk, observed in our study, suggests the possibility that increased MPV reflects advanced vascular damage. Even in asymptomatic patients with multiple CV risk factors.

Namely, damaged vascular cells release substances that activate platelets, and increase thrombogenesis and the potential for development of adverse CV events. $(12,13)$ In this light, it is possible to interpret the results of studies that have shown associations between increased MPV with target organ damage in patients with hypertension and diabetes, $(14,15)$ and also in patients with acute coronary syndrome. $(19,20)$ As in acute coronary syndrome, in which rupture of the fibrous cap allows contact of the platelets with the highly thrombogenic necrotic core, $(19,20)$ vascular damage, caused by multiple CV risk factors, enables contact between platelets and thrombogenic substances even in asymptomatic patients. In both cases, the result is platelet activation. Therefore, increased platelet activity primarily appears as a consequence, not a cause of vascular damage.

Previous studies also stress autonomic imbalances - increased sympathetic or decreased parasympathetic nervous system activity - as an important factor in the progression of atherosclerotic diseases, and development of their complications. (21) Moreover, it has been reported that lower heart rate variability (HRV) - which represents a domination of sympathetic activity - is significantly associated with individual CV risk factors $(22,23)$ and higher total CV risk estimated by FRS. (24) The spleen contains approximately one-third of all the body's platelets. (25) These platelets are relatively larger and hemostatically more active than platelets in the systemic circulation, and can be released into the systemic circulation by stimulation of alpha-adrenoceptors. (25) Therefore, it could be speculated that the correlation between MPV and high CV risk, observed in our patients, is a consequence of sympathetic predomination and increased release of the larger, hemostatically more active platelets from the spleen to the systemic circulation.

We revealed a significant positive linear correlation between FRS values with hs-CRP, which is widely accepted as an independent predictor of CV diseases. $(9,11)$ In agreement with our data, a positive correlation between hs-CRP with calculated 10-year FRS has been previously confirmed. $(9,11)$ Therefore, a positive association between hs-CRP with unadjusted and adjusted calculated 10-year FRS, confirms the meaningfulness of our hypothesis and properly conducted research. Also, this observation suggests hs-CRP is a significantly better marker of CV risk than MPV, and supports the observation that inflammation has an active role in the initiation and progression of atherosclerotic disease. (10)

Study limitations.

We acknowledge the following limitations in this study. This was a cross-sectional rather than a prospective study. Also, the blood sample from each participant in the study was taken only once. Therefore, we were unable to investigate how temporary changes in levels of serum parameters might impact the FRS and its relation to MPV; also, for the same reasons, we were unable to identify a causal relationship between MPV and FRS.

It is possible that rosuvastatin and losartan exhibit antithrombotic properties independently to changes in the lipid profile or blood pressure. $(26,27)$ Therefore, it is important that not a sin- gle patient included in our study used these drugs. There are no data about the potential effect of the other antihypertensive and hypolipidemic drugs on MPV values. Moreover, all of these drugs have been used in previous studies in which positive associations between MPV and single CV risk factors were observed.

Also, we eliminated potential technical factors which could have affected our results. EDTA, used for anticoagulation of whole blood prior to automated cell counting, may increase MPV due to platelet swelling. $(28,29)$ However, it has been confirmed that MPV can be measured accurately by using EDTA and citrate if the analysis is performed within $1 \mathrm{~h}$ of sampling. $(28,29)$ Also, it has been shown that changes in MPV, which reflect platelet sphering and swelling, were greatest between 30 and 60 min in blood stored at ambient temperature. Because, in our study, blood samples were stored at $4^{\circ} \mathrm{C}$ and measurements were performed within 30 min after collecting, using the same device, we are confident in the validity of our laboratory measurements.

Finally, study subjects consisted of patients with a high CV risk referred to us for cardiology assessment for multiple CV risk factors; therefore selection was potentially biased. However, a significant positive (unadjusted and adjusted) association between hs-CRP with calculated 10-year FRS is confirmation that our results were properly taken and processed.

\section{Conclusion}

Linear correlations between hs-CRP with FRS values confirm inflammation as an important mechanism in the initiation and progression of atherogenesis. Correlation of MPV with high total CV risk suggests increased platelet activity primarily as a reflection of advanced atherosclerosis, and possible vascular damage, even in asymptomatic patients. 


\section{ACKNOWLEDGMENTS}

The authors thank Adrijana Livaja and Jadranka Melvan for their technical support. We would also like to thank Assoc. Prof. Damir Fabijanić for his support as mentor on this project.

\section{REFERENCES}

1. Graham I, Atar D, Borch-Johnsen K, Boysen G, Burell G, Cifkova R, et al. European guidelines on cardiovascular disease prevention in clinical practice: full text. Fourth Joint Task Force of the European Society of Cardiology and other societies on cardiovascular disease prevention in clinical practice (constituted by representatives of nine societies and by invited experts). Eur J Cardiovasc Prev Rehabil 2007;14(Suppl 2):S1-113

2. Grundy SM, Balady Gl, Criqui MH, Fletcher G, Greenland P, Hiratzka LF, et al. Primary prevention of coronary heart disease: guidance from Framingham: a statement for healtcare professionals from the AHA Task Force on Risk Reduction. Circulation 1998;97:1876-87.

3. Chu SG, Becker RC, Berger PB, Bhatt DL, Eikelboom JW, Konkle B, et al. Mean platelet volume as predictor of cardiovascular risk: a systematic review and meta-analysis. J Thromb Haemost 2010;8:148-56.

4. Inanc T, Kaya MG, Yarlioglues M, Ardic I, Ozdogru I, Dogan A, et al. The mean platelet volume in patients with non-dipper hypertension compared to dippers and normotensives. Blood Press 2010;19:81-5.

5. Coban E, Bostan F, Ozdogan M. The mean platelet volume in subjects with impaired fasting glucose. Platelets 2006;17:350

6. Kodiatte TA, Manikyam UK, Rao SB, Jagadish TM, Reddy M, Lingaiah HK, et al. Mean platelet volume in type 2 Diabetes mellitus. J Lab Physician 2012:4:5-9

7. Grotto HZW, Noronha JFA. Platelet larger cell ratio (P-LCR) in patients with dyslipidemia. Clin Lab Haematol 2004;6:347-49

8. Coban E, Ozdogan M, Yazicioglu G, Akcit F. The mean platelet volume in patients with obesity. Int J Clin Practice 2004;59:981-2.

9. Albert MA, Glynn RY, Ridker PM. Plasma Concentration of C-Reactive Protein and the Calculated Framingham Coronary Heart Disease Risk Score. Circulation 2003:108:161-5.

10. Libby P, Ridker PM, Maseri A. Inflammation and Atherosclerosis. Circulation 2002;105:1135-43.

11. Shahin Y, Hatfield J, Chetter I. C-reactive protein and the Framingham coronary risk core in patients newly diagnosed with intermittent claudication: a prospective study. Vasc Endovascular Surg 2012;46:242-5

12. Coppinger JA, Cagney G, Toomey S, Kislinger T, Belton O, McRedmond JP, et al. Characterization of the proteins released from activated platelets leads to localizationof novel platelet in human atherosclerotic lesions. Blood 2004;103:2096-104.

13. Margetic S. Inflammation and haemostasis. Biochem Med (Zagreb) 2012;22:49-62.

14. Ates O, Kiki I, Bilen H. Association of mean platelet volume with the degree of retinopathy in patients with diabetes mellitus. Eur J Gen Med 2009;6:99-102

15. Yarlioglues M, Kaya MG, Ardic I, Dogdu O, Kasapkara HA, Gunturk E, et al. Relationship between mean platelet volume levels and subclinical target organ damage in newly diagnosed hypertensive patients. Blood Press 2011;20:92-7.

16. Kutlucan A, Bulur S, Kr S, Bulur S, Önder E, Aslantaş Y, et al. The relationship between mean platelet volume with metabolic syndrome in obese individuals. Blood Coagul Fibrinolysis 2012;23:388-90.

17. Vazzana N, Santilli F, Sestili S, Cuccurullo C, Davi G. Determinants of increased cardiovascular disease in obesity and metabolic syndrome. Curr Med Chem 2011;18:5267-80

18. Dogan A, Aksoy F, Icli A, Arslan A, Varol E, Uysal BA, et al. Mean platelet volume is associated with culprit lesion severity and cardiac events in acute coronary syndromes without ST elevation. Blood Coagul Fibrinolysis 2012;23:324-30.

19. Thiruvagounder M, Khan S, Sheriff DS. The prevalence of metabolic syndrome in a local population in India. Biochem Med (Zagreb) 2010;20:249-52.

20. Chu H, Chen WL, Huang CC, Chang HY, Kuo HY, Gau CM, et al. Diagnostic performance of mean platelet volume for patients with acute coronary syndrome visiting an emergency department with acute chest pain: the Chinese scenario. Emerg Med J 2011;28:569-74.

21. Dekker JM, Crow RS, Folsom AR, Hannan PJ, Liao D, Swenne CA, et al. Atherosclerosis risk in communities. Low heart rate variability in a 2-minute rhythm strip predicts risk of coronary heart disease and mortality from several causes: the ARIC Study. Circulation 2000;102:1239-44.

22. Kupari M, Virolainen J, Koskinen $\mathrm{P}$, Tikkanen MJ. Short-term heart rate variability and factors modifying the risk of coronary artery disease in a population sample. Am J Cardiol 1993;72:897-903

23. Thayer JF, Yamamoto SS, Brosschot JF. The relationship of autonomic imbalance, heart rate variability and cardiovascular disease risk factors. Int J Cardiol 2010;141:122-31.

24. Yoo CS, Lee K, Yi SH, Kim JS, Kim HC. Association of heart rate variability with the framingham risk score in healthy adults. Korean J Fam Med 2011;32:334-40.

25. Baković D, Pivac N, Eterović D, Palada I, Valic Z, Paukovic-Sekulic B, et al. Changes in platelet size and spleen volume in response to selective and non-selective beta-adrenoreceptor blockade in hypertensive patients. Clin Exp Pharmacol Physiol 2009;36:441-6.

26. Coban E, Afacan B. The effect of rosuvastatin treatment on the mean platelet volume in patients with uncontrolled primary dyslipidemia with hypolipidemic diet treatment. Platelet 2008;19:111-4.

27. Jagroop IA, Mikhailidis DP. Angiotensin II can induce and potentiate shape change in human platelets: effect of losartan. J Hum Hypertens 2000;14:581-5.

28. Dastjerdi MS, Emami T, Najafian A, Amini M. Mean platelet volume measurement, EDTA or citrate? Hematology 2006:11:317-9.

29. Macey M, Azam U, McCarthy D, Webb L, Chapman ES, Okrongly D, et al. Evaluation of the anticoagulants EDTA and citrate, theophylline, adenosine, and dipyridamole (CTAD) for assessing platelet activation on the ADVIA 120 haematology system. Clin Chem 2002;48:891-9. 